\title{
Carences nutritionnelles, oligo-éléments et cheveux. Place de l'ICP-MS
}

\section{Hair and diet assessment. Testing for trace elements by ICP-MS}

Pascal KINTZ*

Laboratoire ChemTox, Illkirch.

*Auteur à qui adresser la correspondance : Pascal Kintz, Laboratoire ChemTox,

3 , rue Grüninger, 67400 Illkirch, France

Tél : 0390400540 - Fax : 0390400541 - E-mail : pkintz@labochemtox.com

(Reçu le 30 janvier 2007 ; accepté après modifications le 12 mars 2007)

\section{RÉSUMÉ}

La beauté physique des cheveux (aspect, couleur, brillance ...) a longtemps été interprété comme un reflet de l'état général d'un individu, et en particulier de son régime alimentaire. À l'inverse, plus récemment et lié au développement d'appareillage sensible et spécifique, de type ICP-MS, il a été proposé d'établir un diagnostic en oligo-éléments à partir des cheveux. Les aliments sont alors considérés comme des signaux chimiques qui peuvent être dépistés à long terme dans les cheveux. L'analyse de la littérature permet de distinguer deux situations où le dosage des oligo-éléments dans les cheveux peut être mis en cuvre. L'évaluation du régime alimentaire et la mesure des carences nutritionnelles sont néanmoins des approches très différentes. Le régime alimentaire correspond à ce qui a été volontairement consommé par l'intéressé (eau, nourriture). Les éventuelles carences nutritionnelles sont liées aux concentrations en oligo-éléments nécessaires pour un métabolisme optimal. L'alimentation peut être très variée et conduire au même état nutritionnel. La méthode de criblage et les concentrations physiologiques établies au laboratoire ChemTox de 32 éléments sur une population de 65 personnes âgées de 4 à 73 ans ont été établies et il semble important que chaque laboratoire dispose de ses propres valeurs de référence. Le criblage et le dosage des

\section{SUMMARY}

The appearance of an individual's hair has long been known to reflect his diet and nutritional status. More recently, due to very sensitive and specific apparatus such as ICP/MS, it has been possible to accurately determine trace elements using hair. Metals detectable in hair can be considered to be either nutritionally necessary or toxic, and can be related to both dietary and non-dietary exposure.

The assessment of dietary intake (food and water intentionally consumed by an individual) and the assessment of nutritional status (degree to which an individual has a certain level of nutrients for optimal metabolic function) are two very different issues and it is essential to distinguish between them.

At ChemTox, the analytical approach to test for 32 elements includes decontamination with water and acetone, hydrolysis with nitric acid and analysis using ICP/MS. It is critical that each laboratory establishes its own reference data.

Hair analysis has not yet proved itself as useful a tool for diet and nutrient assessment as it has for toxicity studies. However, if one acknowledges the limitations of current interpretations, these analyses can still yield valuable information. Its advantages, in terms of availability and ease of sampling, 
oligo-éléments dans les cheveux n'ont pas encore montré à la communauté scientifique un intérêt diagnostic majeur, comme cela est le cas pour les molécules organiques. L'absence de qualificatif adéquat provient essentiellement des trop nombreuses limitations de cette approche. Si la phase analytique est aujourd'hui totalement maîtrisée par l'utilisation en routine de l'ICP-MS, les difficultés majeures sont au niveau de l'interprétation des résultats et à l'absence d'homogénéité dans les conclusions rendus à partir du même échantillon. Dans ces conditions, et en particulier du fait de la facilité de prélèvement et de conservation des cheveux, ce type d'investigation pourrait au mieux permettre des études épidémiologique sur une population large.

\section{MOTS-CLÉS}

ICP-MS, oligo-éléments, cheveux, carences.

\section{Introduction}

La beauté physique des cheveux (aspect, couleur, brillance ...) a longtemps été interprété comme un reflet de l'état général d'un individu, et en particulier de son régime alimentaire. A l'inverse, plus récemment et lié au développement d'appareillage sensible et spécifique, de type ICP-MS, il a été proposé d'établir un diagnostic en oligo-éléments à partir des cheveux. Les aliments sont alors considérés comme des signaux chimiques qui peuvent être dépistés à long terme dans les cheveux.

Il est connu depuis fort longtemps que les métaux sont détectables dans les cheveux. Leur pouvoir discriminant pour les intoxications à long terme, qu'elles soient accidentelles $(1,2)$ ou criminelles $(3,4)$ n'est plus à démontrer et de nombreux drames écologiques (arsenic ou plomb dans l'eau de boisson, mercure dans les poissons ...) ont été documentés par des études épidémiologiques dont le cheveu constituait la base biologique. En fait, il s'agit surtout de situations impliquant des minéraux toxiques.

L'analyse de la littérature permet de distinguer deux situations où le dosage des oligo-éléments dans les cheveux peut être mis en oeuvre. L'évaluation du régime alimentaire et la mesure des carences nutritionnelles sont néanmoins des approches très différentes. Le régime alimentaire correspond à ce qui a été volontairement consommé par l'intéressé (eau, nourriture). Les éventuelles carences nutritionnelles sont liées aux concentrations en oligo-éléments nécessaires pour un métabolisme optimal. L'alimentation peut être très variée et conduire au même état nutritionnel. Parmi les oligo-éléments d'importance, il faut mentionner le zinc, le cuivre, le manganèse, le chrome et le sélénium.

L'objet de cet article est de présenter la méthode de criblage du laboratoire et de discuter les aspects scientifiques et commerciaux de l'analyse des oligoéléments dans les cheveux. ensure that many will continue to investigate how far we can reconstruct an individual's dietary and nutritional history from studying the hair.

\section{KEY-WORDS}

ICP-MS, trace elements, hair, diet, nutritional status.

\section{Matériels et méthodes Échantillons}

Les cheveux témoins ont été coupés au ciseau chez 65 personnes, âgées de 4 à 73 ans, vivant en Alsace et n'ayant pas de pathologie particulière. Le prélèvement a été conservéà température ambiante, dans une enveloppe jusqu'au moment de l'analyse.

La conservation dans une feuille de papier aluminium doit être évitée et le kit de prélèvement avec calque doit être privilégié (figures 1 et 2).

Les cheveux doivent être correctement orientés racineextrémité avec une ficelle, la longueur et la couleur soigneusement notées.

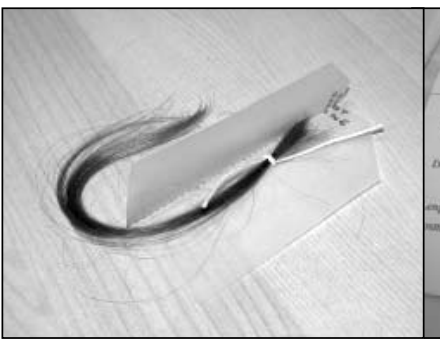

Figure 1 : Mèche de cheveux et support en calque.

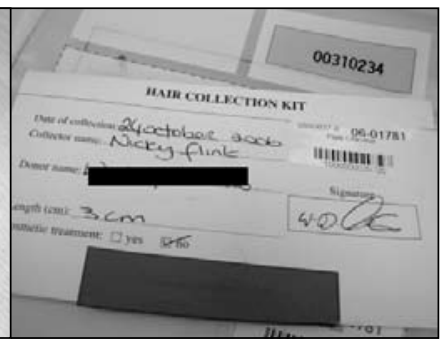

Figure 2 : Kit de prélèvement et de conservation des cheveux.

\section{Solvants et réactifs}

L'acétone, de qualité HPLC, provient de Carlo Erba (Val de Reuil, France). L'acide nitrique SUPRAPUR à $65 \%$ et le Triton X-100 ont été fournis par Merck (Darmstadt, Allemagne). Les solutions de référence ont été achetées chez IV labs ou Merck. Le rhodium, utilisé comme étalon interne provient de chez Merck.

La solution diluant cheveux est constituée de : $100 \mu \mathrm{L}$ d'étalon interne (rhodium à $10 \mathrm{mg} / \mathrm{L}$ ), $100 \mu \mathrm{L}$ de Triton $\mathrm{X}-100,10 \mathrm{~mL}$ d'acide nitrique $65 \%$, qsp $1000 \mathrm{~mL}$ d'eau bi-distillée. 


\section{Préparation des échantillons}

L'échantillon de cheveux est lavé pendant $5 \mathrm{~min}$ à l'eau tiède $\left(40{ }^{\circ} \mathrm{C}\right)$ à raison de $10 \mathrm{~mL}$ pour $100 \mathrm{mg}$ de cheveux, puis séché entre 2 feuilles de papier absorbant. Il est ensuite décontaminé par de l'acétone ( $5 \mathrm{~min}$ ), puis à nouveau séché. Vingt cinq mg de cheveux sont alors mis à hydrolyser dans $250 \mu \mathrm{L}$ d'acide nitrique $65 \%$ pendant $60 \mathrm{~min}$ à $70{ }^{\circ} \mathrm{C}$. Les échantillons et les étalons sont dilués au 1/40 dans le diluant cheveux, puis analysés par ICP-MS.

\section{Analyses ICP-MS}

Trente deux éléments sont dosés par ICP-MS sur un système Thermo Electron X7 (Waltham, MA). La radio-fréquence du générateur est à $1,4 \mathrm{~kW}$. Les débits du plasma d'argon sont : nébulisation $0,95 \mathrm{~L} / \mathrm{min}$, plasmagène $13 \mathrm{~L} / \mathrm{min}$ et auxiliaire $0,90 \mathrm{~L} / \mathrm{min}$.

A chaque série d'analyse, le laboratoire réalise une gamme de calibration en 6 points et vérifie sur une large plage (encadrant les concentrations physiologiques) la linéarité. Très peu de dérive a été observée depuis 3 ans et les coefficients de corrélation sont généralement supérieurs à 0,999 . Les limites de quantifications sont données dans le tableau 1.

Le laboratoire participe à un contrôle de qualité interne (Trace Elements in Human Hair NRM GBW09101, LGC Promochem, Molsheim, France) et à un contrôle de qualité externe (3 séries par an, Centre de Contrôle du Canada).

\section{Résultats}

La procédure décrite ci-dessus permet le criblage et le dosage simultané de 32 éléments.

Les concentrations physiologiques sur une population de 65 personnes vivant en Alsace sont données dans le tableau 1. Ces concentrations sont très voisines de celles publiées par Goulléetcoll (5).L'ensemble des paramètres peut être obtenu en une seule analyse, ce qui fait de l'ICP-MS un excellent outil de criblage. A l'exclusion du nickel, toutes les limites de quantifications sont de l'ordre du pg/mg, permettant de diminuer le cas échéant la prise d'essai (cheveux de nouveau-né, échantillon à caractère historique). Il apparaît que le couplage ICPMS constitue un progrès réel dans l'établissement des valeurs de référence (6). En particulier, il était possible de mésestimer la concentration de certains éléments en très faible quantité, de l'ordre de quelques $\mathrm{pg} / \mathrm{mg}$ et qui correspondait à la limite de détection / quantification des méthodes plus anciennes et donc moins sensibles. Le tableau 2 compare nos concentrations normales avec celles publiées par Goullé et coll (5) et démontre une bonne concordance.
Tableau I : Limites de quantifications (LQ) et concentrations physiologiques de 32 éléments dans les cheveux (par ordre croissant de masse atomique).

\begin{tabular}{|c|c|c|}
\hline Éléments & $\begin{array}{c}\text { LQ } \\
\text { ng/mg }\end{array}$ & $\begin{array}{l}\text { Concentrations physioloiques } \\
\text { ng/mg }\end{array}$ \\
\hline Lithium & 0,019 & $<0,051$ \\
\hline Bérylium & 0,013 & $<0,014$ \\
\hline Bore & 0,02 & $0,13-2,49$ \\
\hline Aluminium & $<0,001$ & $1,0-23,7$ \\
\hline Vanadium & 0,002 & $0,02-0,13$ \\
\hline Chrome & 0,013 & $0,17-0,55$ \\
\hline Manganèse & 0,012 & $0,014-0,90$ \\
\hline Cobalt & 0,002 & $0,009-0,36$ \\
\hline Nickel & 0,541 & $<0,54-2,5$ \\
\hline Cuivre & 0,031 & $8,3-112$ \\
\hline Zinc & $<0,001$ & $109-580$ \\
\hline Gallium & 0,002 & $<0,007$ \\
\hline Germanium & 0,003 & $<0,020$ \\
\hline Arsenic & 0,036 & $0,036-0,16$ \\
\hline Sélénium & $<0,001$ & $0,18-1,3$ \\
\hline Rubidium & 0,013 & $0,013-0,1$ \\
\hline Strontium & 0,007 & $0,6-14,4$ \\
\hline Molybdène & 0,002 & $0,005-0,056$ \\
\hline Palladium & 0,002 & $0,002-0,032$ \\
\hline Argent & 0,002 & $0,02-1,7$ \\
\hline Cadmium & 0,001 & $0,003-0,3$ \\
\hline Etain & 0,002 & $0,017-2,9$ \\
\hline Antimoine & $<0,001$ & $<0,063$ \\
\hline Tellure & 0,004 & $<0,004$ \\
\hline Baryum & $<0,001$ & $0,24-5,3$ \\
\hline Tungstène & $<0,0001$ & $<0,005$ \\
\hline Platine & $<0,0001$ & $<0,002$ \\
\hline Mercure & 0,022 & $0,044-1,74$ \\
\hline Thallium & $<0,0001$ & $<0,002$ \\
\hline Plomb & 0,005 & $0,15-6,13$ \\
\hline Bismuth & $<0,0001$ & $0,001-0,07$ \\
\hline Uranium & $<0,001$ & $0,002-0,075$ \\
\hline
\end{tabular}

Tableau II : Comparaison des valeurs normales entre notre étude et celle de Goulé et al (5).

\begin{tabular}{|c|c|c|}
\hline Éléments & $\begin{array}{c}\text { Goullé (5) } \\
\text { n=45 sujets }\end{array}$ & $\begin{array}{c}\text { notre étude } \\
\mathbf{n = 6 5} \text { sujets }\end{array}$ \\
\hline Chrome & $0,11-0,52 \mathrm{ng} / \mathrm{mg}$ & $0,17-0,55 \mathrm{ng} / \mathrm{mg}$ \\
\hline Cuivre & $9-61,3 \mathrm{ng} / \mathrm{mg}$ & $8,3-112 \mathrm{ng} / \mathrm{mg}$ \\
\hline Zinc & $129-209 \mathrm{ng} / \mathrm{mg}$ & $109-580 \mathrm{ng} / \mathrm{mg}$ \\
\hline Sélénium & $0,37-1,37 \mathrm{ng} / \mathrm{mg}$ & $0,18-1,3 \mathrm{ng} / \mathrm{mg}$ \\
\hline
\end{tabular}

\section{Discussion}

Le choix des cheveux comme matrice biologique d'investigation s'explique facilement. C'est un prélèvement presque toujours disponible (les poils pubiens peuvent être une alternative, mais doivent faire l'objet d'une décontamination plus rigoureuse), facile à prélever (sans douleur et sans intrusion privée) et surtout 
facile à stocker à température ambiante et à envoyer par la Poste dans une enveloppe ou un kit de prélèvement. Avec une vitesse de pousse d'environ $1 \mathrm{~cm}$ par mois, c'est en outre un excellent calendrier historique.

A tous ces paramètres favorables, s'opposent un certain nombre d'inconvénients (7), qui ont fait l'objet de travaux rigoureux pour aboutir à une certaine forme de discréditation dans le milieu scientifique et en particulier en biologie. Les cheveux, oui pour les substances organiques (stupéfiants, médicaments, substances de la performance, pesticides ...), mais certainement pas pour les minéraux... Tel est le postulat communément accepté aujourd'hui !

Les problèmes souvent mentionnés sont les suivants : - contamination externe (avec une impossibilité à éliminer les polluants)

- absence de corrélation entre régime alimentaire ou carence nutritionnelle et signal chimique mesuré

- variation endogène des concentrations mesurées

- vitesse de pousse des cheveux et influence sur les concentrations.

Puisque exposé à l'air ambiant, le cheveu est propice à des contaminations, soit de type endogène par la sueur ou les secrétions sébacées, soit de type exogène par les poussières, les fumées et les traitements cosmétiques. Plus on s'éloigne de la racine, plus le cheveu est ancien et donc susceptible d'être contaminé, en particulier s'il est endommagé. Certains auteurs ont rapporté une corrélation entre la concentration en oligo-éléments et la distance du segment analysé au scalp (8). Il a été montré que les shampooings peuvent modifier à la baisse les concentrations de zinc, cuivre et d'un élément majeur le magnésium (9), tandis que ceux qui sont enrichis en sélénium, cuivre ou zinc (proposés pour renforcer la vitalité des cheveux) peuvent au contraire majorer artificiellement ces concentrations (10). Les traitements cosmétiques (coloration, décoloration, permanente ...), tout comme pour les molécules organiques peuvent altérer le contenu des cheveux (11).

Plusieurs études doses-concentrations dans les cheveux ont montré des résultats discordants. Certains auteurs ont trouvé une corrélation, d'autres pas. $\mathrm{Si}$ une supplémentation en sélénium conduit à une augmentation des concentrations dans les cheveux, il n'en est rien dans le sang (12), ce qui conduit à des difficultés d'interprétation. Par ailleurs, des supplémentations en zinc et cuivre sont sans influence sur les concentrations capillaires (13). Il faut donc reconnaître que les concentrations mesurées ne sont pas que le reflet de l'alimentation, mais dépendent également d'autres facteurs. Il a été montré que les concentrations en cuivre étaient sensiblement les mêmes chez des humains avec un régime alimentaire équilibré et chez les malnutris (14).
Dans ces conditions, il est admis que les concentrations en oligo-éléments ne permettent pas d'établir une éventuelle carence nutritionnelle d'un individu donné et qu'elles peuvent tout au plus être considérées comme un indicateur au niveau de toute une population.

Les concentrations en oligo-éléments dans les cheveux dépendent de nombreux facteurs, comme le sexe, l'âge, la race, la couleur et la région anatomique de prélèvement. Ces paramètres ont probablement plus d'influence que le régime alimentaire. Les cycles de la vie et les changements hormonaux peuvent modifier les concentrations des oligo-éléments. De même, les traitements contraceptifs et la grossesse sont connus pour altérer les concentrations en cuivre (15). Des résultats contradictoires ont été publiés à propos de l'influence de la couleur des cheveux sur les concentrations (16), sans qu'il soit possible d'établir un consensus. Chez un même individu, le manganèse semble être plus concentré dans les cheveux noirs que blancs, mais ce n'est pas le cas pour le zinc et le cuivre (17).

\section{Le problème particulier des analyses commerciales}

Dès 1990, dans une revue générale, Lamand et al (18) qualifient au mieux de rêve, mais plus sûrement d'escroquerie la réalisation de profils métallographiques. Les auteurs se basaient sur leurs travaux et sur une lecture exhaustive de la littérature. Il faut dire qu'en 1985, Barret (19) dans un article polémique se posait la question:"Commercial hair analysis. Science or scam?". Après avoir prélevé des cheveux sur deux adolescents en bonne santé, l'auteur les a envoyés à 13 laboratoires différents. Les résultats étaient fort discordants, les interprétations totalement différentes et 6 laboratoires ont proposé une supplémentation alimentaire. Au final, l'investigateur concluait que l'analyse du cheveu pour les métaux est non scientifique, inutilement coûteuse et peut être en dehors de la légalité !

D'autres études en Allemagne (19) et aux Etats Unis (20) sont parvenus à des conclusions identiques, reprochant aux différents laboratoires une faible précision, des différences inacceptables et des interprétations fausses.

\section{Conclusion}

Le criblage et le dosage des oligo-éléments dans les cheveux n'ont pas encore montré à la communauté scientifique un intérêt diagnostic majeur, comme cela est le cas pour les molécules organiques (22). L'absence de qualificatif adéquat provient essentiellement des trop nombreuses limitations de cette approche. Si la phase analytique est aujourd'hui totalement maîtrisée par l'utilisation en routine de l'ICP-MS, les difficultés majeures sont au niveau de l'interprétation des résultats et à l'absence d'homogénéité dans les conclusions 
rendus à partir du même échantillon (23). Dans ces conditions, et en particulier du fait de la facilité de prélèvement et de conservation des cheveux, ce type d'investigation pourrait au mieux permettre des études épidémiologiques sur une population large.

\section{Références}

1. Bencko V. Use of human hair as a biomarker in the assessment of exposure to pollutants in occupational and environmental settings. Toxicology $1995 ; 101: 29-39$.

2. Kales S.N., Goldman R.H. Mercury exposure : current concepts, controversies and a clinic's experience. J. Occup. Environ. Med. 2002 ; 44 : 143-154.

3. Kintz P., Goullé J.P., Fornes P., Ludes B. A new series of hair analysis from Napoléon confirms chronic exposure to arsenic. J. Anal. Toxicol. $2002 ; 26: 584-585$.

4. Goullé J.P., Mahieu L., Kintz P. The murder weapon was found in the hair! Ann. Toxicol. Anal. 2005 ; 17 : 243246.

5. Goullé J.P., Mahieu L., Castermant J., Neveu N., Bonneau L., Laine G., Bouige D., Lacroix C. Metal and metalloid multi-elementary ICP-MS validation in whole blood, plasma, urine and hair. Reference values. Forensic Sci. Int. $2005 ; 153: 39-44$.

6. Miekeley N., Dias Carnero M.T., da Silveira C.L. How reliable are human hair reference intervals for trace elements ? Sci. Total Environ. 1998 ; 218 : 9-17.

7. Sukumar A. Factors influencing levels of trace elements in human hair. Rev Environ. Contam. Toxicol. 2002 ; $175: 47-78$.

8. O'Connell T. Hair and nutrient/diet assessment. In : Tobin D.J., ed. Hair in Toxicology. Cambridge : Royal Society of Chemistry, 2005 ; 175-193.

9. Abraham J.L. Trace elements in hair. Lancet 1982 ; 8297 : 554-555.

10. LeBlanc A., Dumas P., Lefebvre L. Trace elements content of commercial shampoos : impact on trace element levels in hair. Sci. Total Environ. 1999; 229 : 121-124.

11. Clanet P., DeAntonio S.M., Katz S.A., Scheiner D.M. Effects of some cosmetics on copper and zinc concentrations in human hair scalp. Clin. Chem. 1982 ; $28: 2450-2451$.

12. Hac E., Krechniak J., Szyszko M. Selenium levels in human plasma and hair in northern Poland. Biol. Trace Elem. Res. 2002 ; 85 : 277-285.

13. Gibson R.S., Yeudall F., Drost N., Mititimuni B.M., Cullinan T.R. Experiences of a community-based dietary intervention to enhance micronutrient adequacy of diets low in animal source foods and high in phytate : a case study in rural Malawian children. J. Nutr. 2003 ; 133 suppl 2 : 3992S-3999S.

14. Bradfield R.B., Cordano A., Baertl J., Graham G.G. Hair copper in copper deficiency. Lancet 1980 ; 8190 : 343344.

15. Vir S.C., Love A.H. Zinc and copper nutriture of women taking oral contraceptive agents. Am. J. Clin. Nutr. 1981 ; 34 : 1479-1483.

16. Sky-Peck H.H. Distribution of trace elements in human hair. Clin Physiol. Biochem. 1990 ; 8 : 70-80.
17. Guillard O., Gombert J., Barriere M., Reiss D., Piriou A. Manganese concentration in the hair of greying ("salt and pepper") men reconsidered. Clin. Chem. 1985 ; 31 : 1251.

18. Lamand M., Favier A., Pineau A. La détermination des oligo-éléments dans les poils et les cheveux : intérêt et limites. Ann. Biol. Clin. 1990 ; 48 : 433-442.

19. Barret S. Commercial hair analysis. Science or scam ? JAMA $1985 ; 254: 1041-1045$.

20. Drasch G., Roider G. Assessment of hair mineral analysis commercially offered in Germany. J. Trace Elem. Med. Biol. 2002 ; 16 : 27-31.

21. Seidel S., Kreutzer R., Smith D., McNeel S., Gilliss D. Assessment of commercial laboratories performing hair mineral analysis. JAMA $2001 ; 285: 67-72$.

22. Morley N., Ford R.P. Hair-element analysis - Still on the fringe. Child Care Health Dev. 2002 ; 28 suppl 1 : 31-34

23. Shamberger R.J. Validity of hair mineral testing. Biol. Trace Elem. Res. $2002 ; 87$ : 1-28. 